\title{
Multi-granularity Deep Local Representations for Irregular Scene Text Recognition
}

\author{
HONGCHAO GAO and YUJIA LI, Institute of Information Engineering, Chinese Academy of Sciences, \\ China and School of Cyber Security, University of Chinese Academy of Sciences, China \\ JIAO DAI, XI WANG, and JIZHONG HAN, Institute of Information Engineering, Chinese Academy \\ of Sciences, China \\ RUIXUAN LI, Institute of Information Engineering, Chinese Academy of Sciences, China and School of \\ Computer Science and Technology, Huazhong University of Science and Technology, China
}

\begin{abstract}
Recognizing irregular text from natural scene images is challenging due to the unconstrained appearance of text, such as curvature, orientation, and distortion. Recent recognition networks regard this task as a text sequence labeling problem and most networks capture the sequence only from a single-granularity visual representation, which to some extent limits the performance of recognition. In this article, we propose a hierarchical attention network to capture multi-granularity deep local representations for recognizing irregular scene text. It consists of several hierarchical attention blocks, and each block contains a Local Visual Representation Module (LVRM) and a Decoder Module (DM). Based on the hierarchical attention network, we propose a scene text recognition network. The extensive experiments show that our proposed network achieves the state-of-the-art performance on several benchmark datasets including IIIT-5K, SVT, CUTE, SVT-Perspective, and ICDAR datasets under shorter training time.
\end{abstract}

\section{CCS Concepts: • Computing methodologies $\rightarrow$ Computer vision; $\bullet$ Computer systems organization $\rightarrow$ Redundancy; $\bullet$ Image representations; $\bullet$ Scene text recognition;}

Additional Key Words and Phrases: Scene text recognition, attention mechanism, convolutional neural network

\section{ACM Reference format:}

Hongchao Gao, Yujia Li, Jiao Dai, Xi Wang, Jizhong Han, and Ruixuan Li. 2021. Multi-granularity Deep Local Representations for Irregular Scene Text Recognition. ACM/IMS Trans. Data Sci. 2, 2, Article 15 (March 2021), 18 pages.

https://doi.org/10.1145/3446971

\footnotetext{
This work was supported in part by the National Natural Science Foundation of China under grant 61702502, National Key Research and Development Program of China under grant 2016YFB0800402, National Natural Science Foundation of China under grants U1836204, U1936108, and 61572221, and Major Projects of the National Social Science Foundation under grant 16ZDA092.

Authors' addresses: H. Gao and Y. Li, Institute of Information Engineering, Chinese Academy of Sciences, Hai Dian, Bei Jing, China, 100093, School of Cyber Security, University of Chinese Academy of Sciences, Hai Dian, Bei Jing, China, 100093; email: gaohongchao@iie.ac.cn; J. Dai, X. Wang, and J. Han, Institute of Information Engineering, Chinese Academy of Sciences, Hai Dian, Bei Jing, China, 100093; email: hanjizhong@iie.ac.cn; R. Li (corresponding authors), Institute of Information Engineering, Chinese Academy of Sciences, Hai Dian, Bei Jing, China, 100093, School of Computer Science and Technology, Huazhong University of Science and Technology, Wu Han, China, 430074; email: liruixuan@iie.ac.cn.

Permission to make digital or hard copies of all or part of this work for personal or classroom use is granted without fee provided that copies are not made or distributed for profit or commercial advantage and that copies bear this notice and the full citation on the first page. Copyrights for components of this work owned by others than ACM must be honored. Abstracting with credit is permitted. To copy otherwise, or republish, to post on servers or to redistribute to lists, requires prior specific permission and/or a fee. Request permissions from permissions@acm.org.

(c) 2021 Association for Computing Machinery.

2577-3224/2021/03-ART15 \$15.00

https://doi.org/10.1145/3446971
} 


\section{INTRODUCTION}

Text in scene images often contains rich and valuable information. It can be found on road signs, billboards, and other objects such as cars or telephones. As a branch of the computer vision community, scene text recognition aims to map a cropped scene text image into a set of character sequences or word transcripts. It is one of the most important tools for understanding the content of our world [59]. However, due to the complex environments in the scene text recognition task such as uneven lighting, position changes, and so on, it is a challenge to extract better text representations for this task.

Deep learning has been widely used in many fields, such as computer vision $[34,61]$, natural language processing [38, 60, 63], and database analysis [20, 23, 62]. Recent advances in text representations are driven by the success of deep convolutional neural network (CNN) [27, 33, $37,46,47,49,53]$. Scene text is divided into regular text and irregular text. Regular scene text images are mostly horizontal, and thus text representations captured from a single-granularity visual representation can provide more concise and abstract information for this task. In recent years, researchers have proposed many recognition networks and have shown promising performances on regular scene text recognition [46, 47, 53]. While, in real-world applications, many scene texts are in irregular arrangements (e.g., arbitrarily oriented, curved, and slanted). For example, as shown in Figure 1, the appearances of character " $\mathrm{T}$ " are different in all sub-figures. In this case, roughly capturing text representation from a single-granularity visual representation may lose key information and bring undesired noise. It is difficult to classify scene text into regular or irregular text. Consequently, it is challenging to capture a comprehensive text representation for text recognition from a single-granularity visual representation.

The methodology of obtaining useful representations for specific tasks from multiple granularity representations is widely used in computer vision, such as object detection [29, 31], image classification [10, 42], visual question answering [35], and scene text detection [51]. In this work, we verify that text representations captured from multi-granularity visual representations are also suitable for scene text recognition. Nevertheless, most of the recent recognition networks capture text representations only from a single-granularity visual representation.

Currently, there are two groups of irregular methods: rectification based [33, 37, 47, 49] and attention based [5, 6, 27, 28, 57]. The former tries to use the Spatial Transformer Network (STN) [16] to rectify the irregular text first and then take the rectified result as the input of the scene text recognition network. Although rectifying irregular text can facilitate subsequent feature extraction, STN will increase the computational cost. Especially, it is hard to optimize the STN-based method without human-labeled geometric ground truth. The latter uses attention mechanism to focus on the important regions on visual representation to recognize the current character. However, most of the above methods obtain text representation only from a single-granularity visual representation and rely on character-level or pixel-level annotations or sophisticated designs.

To solve the above problems, based on the local similarity of scene images, we propose a Local Visual Representation Module (LVRM) to capture the local representation from current visual representation (specifically, the feature map extracted by CNNs). Local similarity exists between adjacent pixels in natural scene images. LVRM utilizes adjacent pixels to represent current pixels and captures the local representation by means of self-representation between adjacent pixels or (vectors in feature maps). Compared with the kernel used in CNN, our proposed LVRM does not need to learn a fixed number of descriptor that can only represent a fixed pattern, but to extract local representation through self-representation between adjacent pixels.

When recognizing scene text images, the local similarity still exists between adjacent vectors in visual representation extracted by CNNs. Here, local similarity refers to the same semantic 


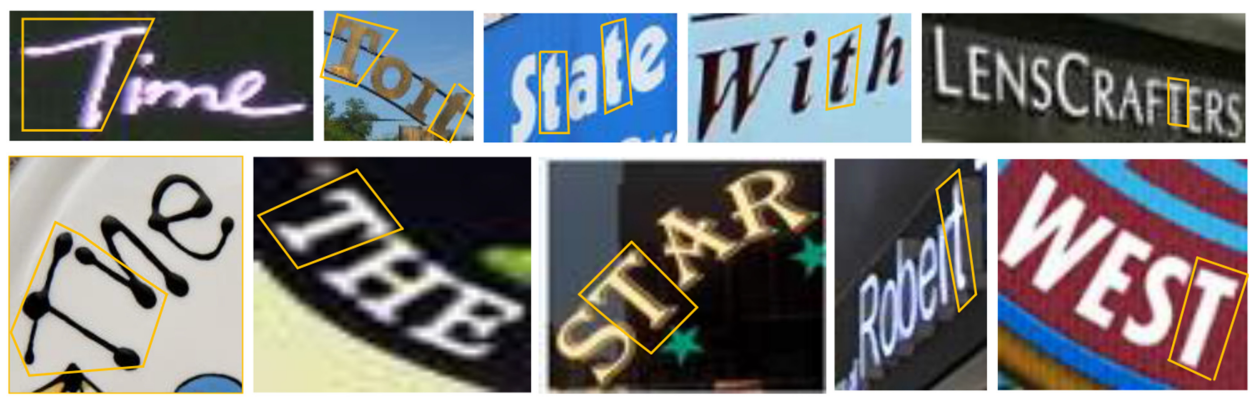

Fig. 1. Examples of irregular (distorted or curved) text in scene images. We illustrate the appearances of character "T."

(character) information that adjacent vectors share. Local representation can be interpreted as a character descriptor, while each vector in visual representation only contains partial information about its corresponding character. For each vector, our proposed LVRM utilizes its adjacent vectors to represent it. Consequently, applying LVRM on visual representation, each vector contains the whole text information about the corresponding character. At the same time, LVRM can also avoid the interference from complex background.

Based on LVRM, we propose a hierarchical attention network to capture multi-granularity deep local representations from multiple visual representations and fuse the captured local representations to obtain the final comprehensive text sequence. Further, we combine a backbone network that is applied for extracting multiple visual representations with the hierarchical attention network to form scene text recognition architecture.

We summarize our contributions as follows:

- We propose a local visual representation module (LVRM) to capture local text representation of characters.

- We propose a hierarchical attention network to capture comprehensive text representations from multi-granularity visual representations.

- We integrate the hierarchical attention network into the recognition network. The whole architecture can be directly trained end-to-end without any character-level annotations.

- Our proposed recognition network achieves excellent results on both regular and irregular scene text recognition with less training time.

\section{RELATED WORK}

The goal of text recognition task is converting a scene text image into a string. Recently, with the great success of deep neural networks on image classification [15] and neural machine translation $[8,52]$, lots of approaches for scene text recognition have been proposed.

Traditionally, methods for scene text recognition are mainly based on bottom-up recognition. Early works mainly divide developed bottom-up recognition into three steps: first extracting lowlevel features for individual character region; then detecting and recognizing one-by-one; finally, integrating these characters into a full word based on a set of heuristic rules or a language model $[41,58]$.

Regular text recognition: Many methods [12, 30, 46, 52] solved this problem as a sequence labeling task, where the input sequence was regarded as a group of text representations of image patches to be recognized, and they directly predicted the entire text from the original image without detecting the character regions. For example, Shi et al. [46] employed Long Short Term 
Memory (LSTM) to model the sequences without explicit character region and used a Connectionist Temporal Classification (CTC) [13] layer to decode the sequences. Wang et al. [53] combined a gated recurrent convolutional neural network with CTC for recognizing regular text. Alternatively, Jaderberg et al. [18] designed a 90k-class CNN with structured output layer for constrained recognition in which each class represented an English word. But it is difficult to apply to other text recognition tasks such as telephone numbers.

Irregular text recognition: The recognition methods of irregular text can be divided into two groups: one group is to rectify the text and then recognize it [19, 37, 47, 49]. Shi et al. [49] first used STN to rectify oriented or curved text, and then used the sequence to sequence model to recognize the rectified results. Liu et al. [33] proposed recognizing and rectifying individual character instead of whole text image. The other group is based on attention mechanism, which focuses on the attention on the right patches of character in images [5, 6, 27, 28, 57]. For example, Cheng et al. [6] used LSTM to encode the image text in four directions, which fully considered the distortion of irregular text and thus extracted better text representations. Cheng et al. [5] found that there was an "attention drifts" problem in traditional attention mechanism and proposed a focusing attention network to solve the problem, which combined an attention network for character recognition and a focusing network. Reference [28] used two LSTM models as 2-D attention model to give the glimpse where to attend. Belonging to the latter, in our proposed attention network, an LVRM gives the glimpse by using the high correlation between adjacent vectors in visual representation, and a hierarchical attention network responses for capturing multi-granularity text representations.

Local representation: The local representation, which is obtained from the information on adjacent pixels, is used for representing the scene image. Many methods are proposed to capture local representations, from the traditional feature descriptors, such as HOG [7] and LBP (Local Binary Patterns) [43], to recently commonly used deep feature descriptor: CNN kernels. The representation can be used for image classification [11, 43, 56], interest point detectors [39], and image denoising [4]. For example, based on the fundamental properties of local image texture, Ojala et al. [43] proposed LBP operator to capture the local image texture. Furthermore, motivated by LBP, $\mathrm{Xu}$ et al. [56] proposed local binary convolution, which comprises a set of fixed sparse pre-defined binary convolutional filters.

\section{MODEL ARCHITECTURE}

The architecture of our recognition network is shown in Figure 2. Overall, the architecture consists of three main components, namely, encoder network, multi-granularity hierarchical attention network, and a transcription layer.

Concretely, given a text image $I$ and its labels $L=\left(l_{1}, \ldots, l_{n}\right)$, where $n$ is the length of labels, we first use a convolutional neural network to extract multi-granularity visual representations of text image $I$. This can be seen as the encoder in the encoder-decoder structure.

Then, we design a hierarchical attention network to obtain comprehensive text representations by fully capturing multi-granularity visual representations. This network consists of three attention blocks. In each block, a local visual representation encoder module and a decoder module are designed equally as an ensemble.

Finally, we concatenate the obtained fixed size text representations, which is the input of the transcription layer.

In the following sections, we will detail the design of our method from the aspects of image feature encoder, hierarchical attention network, and the final fused text representations transcription.

Moreover, Figure 2 shows the recognition network architecture in training phase. In inference phase, we use ResNet [15] to extract multi-granularity representations of input images. Then, we 


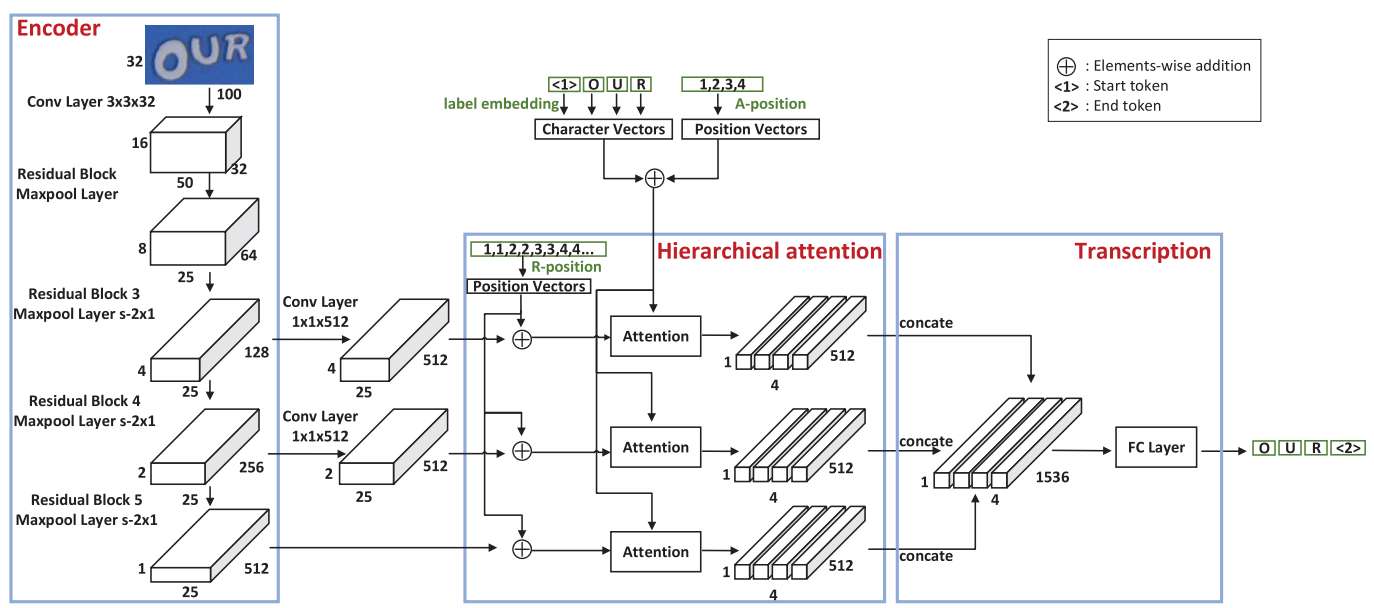

Fig. 2. Overview of the proposed framework. A-position means absolute positional embedding. R-position means relative positional embedding, and we illustrate relative position embedding at stride $2(t=2)$.

use the learned label embedding to convert start token " $\langle 1\rangle$ ” to a vector. This token can be used to capture the text representation of the first real character ("O") of text. After that, we use transcription layer to convert the text representation to character "O." Finally, we use label emdedding to convert " $\langle 1\rangle$ " and "O" to vectors, which are used to capture the text representation of character "U.” Iterate this inference process until predicting the end token " $\langle 2\rangle$.

\subsection{Encoder}

The encoder is responsible for encoding the scene text images into multi-granularity visual representations. We adopt a powerful ResNet-based [15] convolution neural network to obtain multigranularity visual representations of text image. Before entering the network, the image $I$ needs to be resized to fixed size. $F_{w, h, d}^{b}$ is the feature maps produced by the $b$ th residual block and $w, h, d$ represent the width, height, and number of channels of feature maps, respectively.

We use the visual representations from the last three residual blocks. Due to only using convolution and max-pooling operations, $F_{i, j,:}^{b}$ is the feature vector at position $(i, j)$ and represents a receptive field of the input image, which can be considered as the image descriptor for that patch $[46,53]$. For vanilla attention [52] mechanism only decodes 1-D feature sequences that respond to 2 -D visual representations. Thus, we first reshape the extracted 3-D visual representations into a 2-D tensor $F_{w \times h, d}^{b}$. Then, we slice it from left to right by column to form the final visual representation $X^{b}=\left\{X_{1}^{b}, \ldots X_{i}^{b}, \ldots X_{w \times h}^{b}\right\}\left(X_{i}^{b}=F_{i,:}^{b}\right.$ ), where the ":" means all the number of channels of the feature maps are considered.

\subsection{Hierarchical Attention Network}

We propose a hierarchical attention network to capture comprehensive text representations, which is the most critical part of recognition architecture. It consists of three hierarchical attention blocks and each block is responsible for capturing fixed size text representations from different granularity visual representation. Though having the same size, they contain coarse-to-fine text representations. During training, the inputs to each block are the single visual representation and the concatenation of a start token $(\langle 1\rangle)$ and labels $L$.

The attention block contains two modules, namely, an LVRM, which is used to obtain precise description of characters, and a decoder module (DM), which is used to decode the arbitrary 
granularity visual representations into a fixed size text representation. In this section, we first introduce key components of attention block, including label and positional embedding and dot-product attention mechanism. Then, we give detailed description of the two modules.

3.2.1 Key Components. Label and positional embedding: As in the common recurrent based model, labels are embedded in the beginning of the decoder. In our network, we use learned embedding to convert a character $l_{i}$ in $L$ to a character vector $c_{i} \in \mathbb{R}^{d}$ and $d$ represents the dimension of input vector. To make use of positional information, we use positional embedding to convert the absolute position of $l_{i}$ in $L$ to $U_{i} \in \mathbb{R}^{d_{p}}$. In this work, we use sine and cosine functions of different frequencies to encode the $i$ th position $\left(U_{i}=P M(i)\right)$ :

$$
\begin{gathered}
P E_{(i, 2 j)}=\sin \left(i / 10000^{2 j / d}\right) \\
P E_{(i, 2 j+1)}=\cos \left(i / 10000^{2 j / d}\right) \\
P M(i)=P E_{(i,:)},
\end{gathered}
$$

where $i$ is the position, $j$ is the dimension, and ":" means all the number of dimensions. Both are combined to obtain input characters sequence vectors $L=\left(c_{1}+p_{1}, \ldots, c_{n}+p_{n}\right)$.

Dot-product attention mechanism is utilized in DM modules to generate comprehensive representations of the next character by focusing on a significant part of text sequence automatically. A special case is self-attention mechanism, if two sequences are from the same representation $(X=Z)$, which has shown promising empirical results in various tasks [12, 52]. We refine this mechanism and utilize it both LVRM and DM modules to capture representations that are more beneficial to text representation.

In dot-product attention mechanism, we use three learnable weight matrices to project a query and a set of key-value pairs to an output, where the query, keys, values, and output are all vectors. Three learnable weight matrices are $\theta_{q} \in \mathbb{R}^{d \times d_{s}}, \theta_{k} \in \mathbb{R}^{d \times d_{s}}$, and $\theta_{v} \in \mathbb{R}^{d \times d}$, where $d$ and $d_{s}$ are the dimension(channel) of the input vectors and the projected subspace, respectively.

Given two sequences $X=\left\{X_{1}, \ldots X_{i}, \ldots X_{m}\right\}$ and $Z=\left\{Z_{1}, \ldots Z_{j}, \ldots Z_{n}\right\}$, where $m, n$ are the length of sequences, we use weight matrices $\theta_{q}$ to project $i$ th vector in $X\left(X_{i}\right)$ to a query vector $Q_{i}\left(Q_{i}=\theta_{q} X_{i}\right)$ and use two weight matrices to project $j$ th vector in $Z\left(Z_{j}\right)$ to a key vector $K_{j}$ $\left(K_{j}=\theta_{k} Z_{j}\right)$ and a value vector $V_{j}\left(V_{j}=\theta_{v} Z_{j}\right)$.

To compute attention scores and attention outputs, we apply scaled dot-product between the query vector and the key vector. The attention score is calculated as follows:

$$
a_{j}=\frac{Q_{i} \cdot K_{j}}{\sqrt{d}},
$$

where $\sqrt{d}$ is a scaling factor that is used to overcome large values of dot products that push softmax function into extremely small gradients regions [52]. Then, we use softmax function to transform the attention score to obtain the corresponding weight assignment:

$$
\hat{a}_{j}=\frac{a_{j}}{\sum_{i=1}^{i=n} \exp \left(a_{i}\right)} .
$$

After that, the attention output vector $\hat{X}_{i} \in \mathbb{R}^{d \times d}$ for $X_{i}$ is a weighted sum over the value vectors $V_{j}$ :

$$
\hat{X}_{i}=\sum_{j=1}^{j=n} \hat{a_{j}} \cdot V_{j} .
$$

Finally, we add the fourth parameter matrix $\theta_{o} \in \mathbb{R}^{d \times d}$ on the weighted sum vector to capture richer representations of the current vector. 


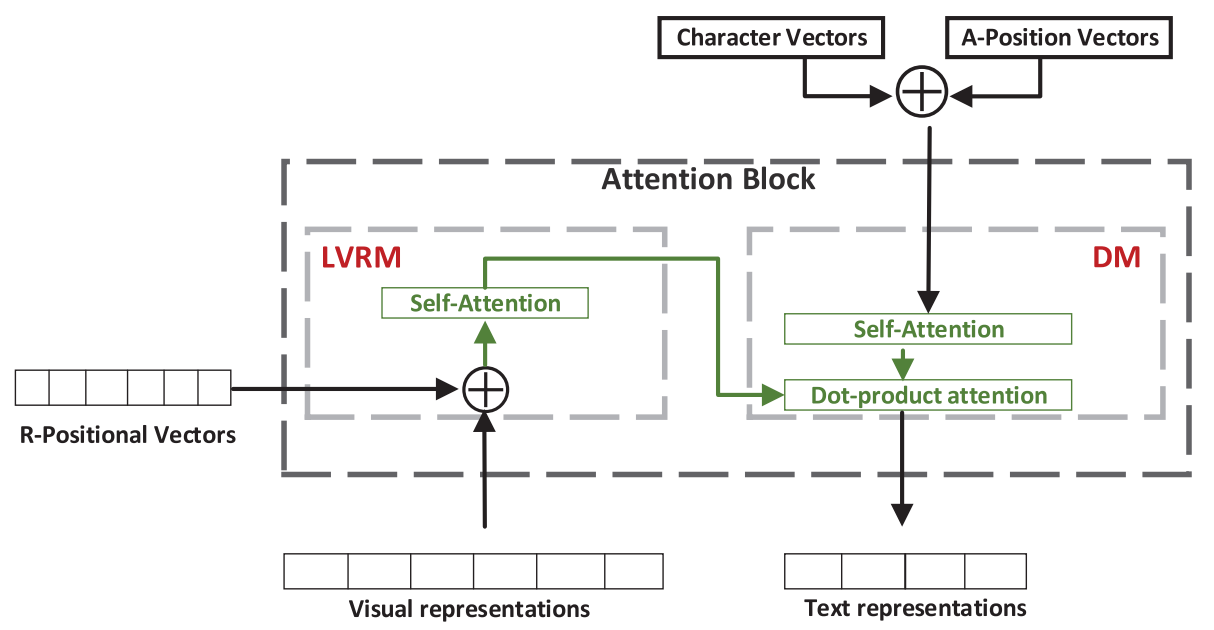

Fig. 3. The attention block.

3.2.2 Attention Block. As shown in Figure 3, the block contains an LVRM and a DM. For the former module, we first inject relative positional information into visual representation and reshape it, and then use a self-attention layer to further capture visual representations. For the latter, we first combine label and absolute positional embeddings to give the fundamental character representations, then use a self-attention layer to obtain representation further, and finally use a dot-product attention layer to capture text sequence.

Local visual representation module (LVRM): Local similarity exists between adjacent pixels in natural scene images. The encoder only consists of convolutional layers and pooling layers. Thus, in the extracted visual representations (feature maps), the local similarity still exists between adjacent vectors. Specifically, for scene text recognition, in visual representations $X^{b}$ provided by encoder, each character region corresponds to a range of continuous image patches that is mapped to be adjacent vectors. Consequently, these adjacent vectors represent the same character and have higher correlation. It other words, each vector in $X^{b}$ only contains part of character information.

Based on this property, for each vector in visual representations $X^{b}$, our proposed LVRM uses its adjacent vectors to represent it. With this module, the character information can flow between adjacent vectors. In this way, each vector obtains a complete representation of its corresponding character based on the partial information contained in its neighbors. In addition, each kernel (neuron) in the convolutional layer, which can also be regarded as local feature descriptor, is used for describing the feature of its receptive field. Compared with CNN, LVRM does not need to learn a fixed number of descriptors that can only represent a fixed pattern, but only to extract local representation through self-representation between adjacent pixels.

Our proposed LVRM is based on self-attention mechanism. If we directly use the vanilla attention [52] for scene text recognition, the input is the element-wise addition of visual representation $X^{b}$ and the absolute positional embeddings $U$. Here, $U_{i}$ only provides the order information of $i$ th vector and ignores the high correlations between adjacent vectors. We assume that adjacent vectors are in the same char-level position and have the same relative position:

$$
U_{i}^{t}=P M(f(i, t)),
$$

where $f(i, t)=\lceil i / t\rceil$ is the $\lceil i / t\rceil$-th semantic segment.

If we inject this information in attention module, it can give the module a temporal clue or "bias" about how information should be gathered, i.e., where to attend when capturing the current 
character. However, there is no way to determine in advance which adjacent vectors represent the same character.

To solve this problem, we inject multiple char-level positional embeddings in the LVRM. Different strides correspond to different partitions that are different relative positions. Therefore, the same representation of absolute position $i$ can be represented by multiple relative positions $f(i, t)$. We encode each relative position $f(i, t)$ into vector $U_{i}^{t}$ and concatenate these vectors as the final relative positional embeddings. Formulated, we define multiple char-level positional embeddings as follows:

$$
U_{i}=\operatorname{Concat}\left(U_{i}^{2}, U_{i}^{3}, U_{i}^{4}, U_{i}^{5}\right) .
$$

Given a sequence that contains $s$ vectors, we first split it into $\lceil s / t\rceil$ segments according to stride $t \in\{2,3,4,5\}$ and assume that each segment represents similar semantic information. Then, we generate the char-level positional embeddings using Equation (6). Finally, we concatenate these as the relative positional embeddings of the sequence.

The actual input to LVRM is the element-wise addition of the visual representation and its relative positional embedding (we set $d_{p}=d / 4$ ). In this way, our proposed module can capture more fundamental text representation of each character. Concretely, given the captured visual representation $F_{w, h, d}^{b}$, we first generate the multiple char-level positional embeddings $U_{i}$ based on the width $w(w=s)$ of $F$. Then, we perform the element-wise addition on each row of visual representation $F_{;, h,:}^{b}$. Finally, we use this representation as the input of LVRM.

Decoder module (DM): The function of the DM is to generate a fixed size text representation from different granularity visual representations that are provided by LVRM. As shown in Figure 3, the DM has two sub-layers: a self-attention sub-layer and a dot-product attention sub-layer.

Given the embedded label sequence $L$, we first use a self-attention module to capture richer representations. The input of this sub-layer is the element-wise addition of the absolute positional embeddings and label embeddings. In sub-layer, a masked self-attention is used to encode the input to prevent leftward information. The following sub-layer is dot-product attention, which is used to capture representations of the next character by focusing on a significant part of text sequence. Here, a query vector $Q_{i}$ is from the previous self-attention sub-layer, key-value pairs vectors are obtained by projecting the local visual representation vector, and we also add a learnable parameter matrix to capture more comprehensive representations. We implement this sub-layer as one-dimensional fully connected convolutional operations.

\subsection{Transcription}

As shown in the Figure 2, each attention block $b$ outputs a fixed size text representation $\hat{X}^{b}$. We need to use the transcription layer to convert these text representations to characters. This layer consists of a fully connected sub-layer and the following softmax sub-layer.

First, we concatenate these representations in the channel dimension as the final text sequence:

$$
R=\operatorname{Concat}\left(\hat{X}^{3}, \hat{X}^{4}, \hat{X}^{5}\right) \text {. }
$$

Then, we use a fully connected layer to obtain the score of each character and use the softmax layer to turn those scores into probabilities:

$$
p\left(y_{k} \mid y_{k-1}, \ldots, y_{1}\right)=\operatorname{softmax}\left(W_{o} R_{k}\right),
$$

where $y_{k-1}, \ldots, y_{1}$ are corresponding history characters, $W_{o} \in \mathbb{R}^{d \times d_{f}}$ and $R_{k}$ are the $k$ th vectors of concatenated representations. The architecture of our recognition network is shown in Figure 2. Overall, the architecture consists of three main components, namely, encoder network, multi-granularity hierarchical attention network, and a transcription layer. 
Concretely, given a text image $I$ and its labels $L=\left(l_{1}, \ldots, l_{n}\right)$, where $n$ is the length of labels, we first use a convolutional neural network to extract multi-granularity visual representations of text image $I$. This can be seen as the encoder in the encoder-decoder structure.

Then, we design a hierarchical attention network to obtain comprehensive text representations by fully capturing multi-granularity visual representations. This network consists of three attention blocks. In each block, a local visual representation encoder module and a decoder module are designed equally as an ensemble.

Finally, we concatenate the obtained fixed size text representations, which is the input of the transcription layer.

In the following sections, we will detail the design of our method from the aspects of image feature encoder, hierarchical attention network, and the final fused text representations transcription.

Moreover, Figure 2 shows the recognition network architecture in training phase. In inference phase, we use ResNet [15] to extract multi-granularity representations of input images. Then, we use the learned label embedding to convert start token " $\langle 1\rangle$ " to a vector. This token can be used to capture the text representation of the first real character ("O") of text. After that, we use transcription layer to convert the text representation to character "O." Finally, we use label emdedding to convert " $\langle 1\rangle$ " and "O" to vectors, which are used to capture the text representation of character "U." Iterate this inference process until predicting the end token " $\langle 2\rangle$. ."

\section{EXPERIMENT}

\subsection{Datasets}

Seven popular benchmarks for scene text recognition are used for performance evaluation.

IIIT5K [40] is collected from the Internet, containing 3,000 cropped word images. In addition, each image is associated with 50 -word and $1 \mathrm{~K}$-word lexicon.

Street View Text (SVT) [54] contains 647 cropped word images that are collected from Google Street View. In addition, each image has a 50 -word lexicon.

ICDAR 2003 (IC03) [36] contains 251 scene images that have bounding boxes of labeled text and 860 cropped images of the words.

ICDAR 2013 (IC13) [22] contains 1,015 cropped text images. For fair comparison, we remove images that contain non-alphanumeric characters. This dataset inherits most data in IC03.

ICDAR 2015 Text (IC15) [21] is generated by a pair of Google Glasses with blurry positioning and focusing. For fair comparison, we discard the images that contain non-alphanumeric characters and finally obtain 1,922 images.

SVT-Perspective (SVTP) [44] contains 645 cropped images and each image has been severely distorted.

CUTE [45] is mainly used for curved text, containing 288 severely distorted images.

We use two training datasets including Synth90K (90K) [18], which contains 9M images, and two-thirds of SynthText (ST) [14], which is a synthetic text dataset. For fair comparison, we do not utilize any training datasets such as SynAdd, the $1.6 \mathrm{M}$-word synthetic data used by References [5, $6,19,55]$ and do not utilize character-level annotations used by Reference [49].

\subsection{Implementation Details}

The ResNet-50 structure proposed in Reference [15] is used to extract the multi-granularity visual representations of scene images. The configurations of ResNet-50 are listed in Table 1. Each gray-scale image is resized to $100 \times 32$. The output of transcription layers is fully connected linear projections with 39 units (26 letters, 10 digits, 3 symbols (start token " $\langle 1\rangle$," end token "〈2〉," 
Table 1. Encoder Network Configurations

\begin{tabular}{l|c|c}
\hline Layers & Out Size & Configurations \\
\hline Block 0 & $32 \times 100$ & $3 \times 3$ conv,$s 1 \times 1$ \\
Block 1 & $16 \times 25$ & {$\left[\begin{array}{c}1 \times 1, \text { conv }, 32 \\
3 \times 3, \text { conv }, 32\end{array}\right] \times 3, s 2 \times 2$} \\
Block 2 & $8 \times 25$ & {$\left[\begin{array}{c}1 \times 1, \text { conv }, 64 \\
3 \times 3, \text { conv, } 64\end{array}\right] \times 4, s 2 \times 2$} \\
Block 3 & $4 \times 25$ & {$\left[\begin{array}{c}1 \times 1, \text { conv, } 128 \\
3 \times 3 \text { conv, } 128\end{array}\right] \times 6, s 2 \times 1$} \\
Block 4 & $2 \times 25$ & {$\left[\begin{array}{l}1 \times 1, \text { conv }, 256 \\
3 \times 3 \operatorname{conv}, 256\end{array}\right] \times 6, s 2 \times 1$} \\
Block 5 & $1 \times 25$ & {$\left[\begin{array}{l}1 \times 1, \text { conv }, 512 \\
3 \times 3, \text { conv }, 512\end{array}\right] \times 3, s 2 \times 1$} \\
\hline
\end{tabular}

Each block is a residual network block. $s$ stands for stride of the first convolutional layer in a block. "Out Size" is feature map size for convolutional layers (height $\times$ width).

and padding token " $\langle 0\rangle$ ”), which is used to transform the decoder output into prediction probabilities over character classes. We utilize standard cross-entropy function to compute the loss of the predicted probabilities w.r.t. the ground-truth at each decoding position. The objective function is defined as follows:

$$
L(\theta)=-\sum_{(I, L) \in D_{\text {train }}} P(L \mid I ; \theta) .
$$

We use the Adam optimizer [24] with the learning rate $\operatorname{lr}=10^{-3}, \beta_{1}=0.9, \beta_{2}=0.99$ and $\epsilon=10^{-8}$, with a decay rate of 0.5 every epoch. Our network is implemented under the Pytorch framework and trained on a computer with an NVIDIA Tesla P100 GPU. The batch size is set to 128 and training is stopped after 6 epochs over the training sets.

There are two evaluating metrics for the recognition performance assessment: word-level recognition rate and normalized edit distance. For word-level recognition rate, the GT string and the predicted string must be exactly the same for the prediction to be correct. In this article, we use the former evaluating metric.

\subsection{Ablation Experiments on Model Components}

The major contribution of our approach is capturing multi-granularity text representations for scene text recognition. To measure the contributions of each component, we progressively remove the captured representations and compare the results. SynthText was originally designed for scene text detection, and it contains some blur and small images. We filter out low-quality images and only keep two-thirds for training. In consideration of allowing researchers to more objectively understand the role of each components of the model, we use Synth90K as training datasets in the ablation study. For all the experiments in this subsection, we use Synth90K as training datasets and use the same training settings.

The ablation experiments about the effects of using different components are listed in Table 2. The performance is the worst when we only use single-granularity visual representations from residual block 5 (Res 5). When we combine Res 5 and Res 4 (the second row), the performance increases by an average of $2.4 \%$. If we use Res 5 and Res 3 (the third row), the performance is $2.9 \%$ higher than only using the single-granularity representations (Res 5). This means using two-granularity representations can effectively improve the accuracy of text recognition. When 
Table 2. Effects of Different Granularity Representations, Relative Positional Embedding, and Self-attention

\begin{tabular}{lll|c|c|ccccccc}
\hline Res 3 & Res 4 & Res 5 & R-position & S-DM & IIIT5K & SVT & IC03 & IC13 & IC15 & SVTP & CUTE \\
\hline & & $\sqrt{ }$ & $\sqrt{ }$ & $\sqrt{ }$ & 79.3 & 80.1 & 89.9 & 90.1 & 66.1 & 70.3 & 63.5 \\
& $\sqrt{ }$ & $\sqrt{ }$ & $\sqrt{ }$ & $\sqrt{ }$ & 83.5 & 82.9 & 91.5 & $\mathbf{9 2 . 7}$ & 68.5 & 72.5 & 64.9 \\
$\sqrt{ }$ & & $\sqrt{ }$ & $\sqrt{ }$ & $\sqrt{ }$ & 84.0 & 82.7 & 91.9 & 92.3 & 68.9 & 73.5 & 66.3 \\
$\sqrt{ }$ & $\sqrt{ }$ & $\sqrt{ }$ & $\sqrt{ }$ & & 81.4 & 80.9 & 90.1 & 90.9 & 66.7 & 72.3 & 64.9 \\
$\sqrt{ }$ & $\sqrt{ }$ & $\sqrt{ }$ & & $\sqrt{ }$ & 84.1 & 82.4 & 92.3 & 91.7 & 69.6 & 74.0 & 66.9 \\
$\sqrt{ }$ & $\sqrt{ }$ & $\sqrt{ }$ & $\sqrt{ }$ & $\sqrt{ }$ & $\mathbf{8 4 . 5}$ & $\mathbf{8 3 . 3}$ & $\mathbf{9 3 . 1}$ & $\mathbf{9 2 . 7}$ & $\mathbf{7 1 . 1}$ & $\mathbf{7 6 . 3}$ & $\mathbf{6 9 . 4}$ \\
\hline
\end{tabular}

"Res $i$ " represents the visual representations captured from $i$ th Residual block. R-position means relative positional embedding. S-DM means with/without self-attention in DM module in Figure 3.

Table 3. Effects of Using Different Attention Heads $(h)$ and Attention Key $\left(d_{k}\right)$ and $\operatorname{Value}\left(d_{v}\right)$ Dimensions

\begin{tabular}{ccc|c|c|c|ccccccc}
\hline Res 3 & Res 4 & Res 5 & $h$ & $d_{k}$ & $d_{v}$ & IIIT5K & SVT & IC03 & IC13 & IC15 & SVTP & CUTE \\
& & $\sqrt{ }$ & 1 & 512 & 512 & 75.1 & 76 & 85.5 & 84.7 & 58.0 & 62.3 & 50.4 \\
& & $\sqrt{ }$ & 8 & 64 & 64 & 79.3 & 80.1 & 89.9 & 90.1 & 66.1 & 70.3 & 63.5 \\
$\sqrt{ }$ & $\sqrt{ }$ & $\sqrt{ }$ & 1 & 512 & 512 & 76.8 & 78.9 & 88.6 & 87.3 & 63.3 & 66.9 & 54.9 \\
$\sqrt{ }$ & $\sqrt{ }$ & $\sqrt{ }$ & 4 & 128 & 128 & 81.0 & 79.9 & 89.1 & 88.4 & 64.2 & 68.4 & 58.9 \\
$\sqrt{ }$ & $\sqrt{ }$ & $\sqrt{ }$ & 8 & 64 & 64 & $\mathbf{8 4 . 5}$ & 83.3 & $\mathbf{9 3 . 1}$ & $\mathbf{9 2 . 7}$ & $\mathbf{7 1 . 1}$ & $\mathbf{7 6 . 3}$ & $\mathbf{6 9 . 4}$ \\
$\sqrt{ }$ & $\sqrt{ }$ & $\sqrt{ }$ & 16 & 32 & 32 & $\mathbf{8 4 . 5}$ & $\mathbf{8 5 . 0}$ & 92.1 & 92.3 & 70.9 & 74.5 & 65.6 \\
\hline
\end{tabular}

we use Res 3, Res 4, and Res 5, the performance is improved by $1.8 \%$ on average compared with using two-granularity representations. It shows that using multi-granularity representations is very important especially for the irregular text recognition.

In addition, we conduct a comparative experiment on the importance of relative (char-level) positional embedding and self-attention in DM. From the results, we can infer that the char-level relative positional embedding is useful for capturing precise text representation of character and multi-granularity attention network can capture comprehensive text representations for scene text recognition.

The ablation experiments about the effects of using different number of attention heads and the attention key and value dimensions are listed in Table 3. In the case using three visual representations, the performance is the worst when we only use one head $(h=1)$. When we increase the number of heads, the performance improves. In particular, the accuracy is highest when the number of heads is $8(h=8)$, increasing by an average of $7.7 \%$ across all datasets compared to using only one head. It is worth noting that using 8 heads improved by $10 \%$ on SVTP and $14.5 \%$ on CUTE, respectively, compared to just using one head. But when we increased the number of heads to $16(h=16)$, the accuracy rate declined by $0.8 \%$. The reason is that lower dimension in project subspace will result in reducing the credibility of correlation measurement when perform scaledot product attention and consequently leading to the low-quality local representation captured by LVRM.

We can learn that when the dimension of recognition network remains unchanged, adding more attention heads can improve the recognition results. However, the recognition performance also drops off with too many heads.

\subsection{Performance on General Recognition Datasets}

In this section, we show the performance of our method and compare with other recent methods. The datasets are divided into regular datasets and irregular datasets. The regular datasets include 
Table 4. Regular Scene Text Recognition Accuracy on Benchmark Datasets

\begin{tabular}{c|c|c|ccccc}
\hline Type & Methods & ConvNet,Data & IIIT5K & SVT & IC03 & IC13 & Params $\left(\times 10^{6}\right)$ \\
\hline \multirow{6}{*}{ I } & Jaderberg et al. [18] & VGG,90K & - & 80.7 & 93.1 & 90.8 & \\
& Jaderberg et al. [17] & VGG,90K & - & 71.7 & 89.6 & 81.8 & \\
& Shi et al. [46] & VGG,90K & 78.2 & 80.8 & 89.4 & 86.7 & 8.3 \\
& Shi et al. [47]* & VGG,90K & 81.9 & 81.9 & 90.1 & 88.6 & 10.8 \\
& Lee et al. [26] & VGG,90K & 78.4 & 80.7 & 88.7 & 90 & \\
& Wang et al. [53] &,$- 90 \mathrm{~K}$ & 80.8 & 81.5 & 91.2 & - & 4.6 \\
& Shi et al. [49] & VGG,90K & 81.7 & 85.5 & 92.2 & 88.6 & - \\
& Shi et al. [49] & ResNet,90K & 83.2 & $\mathbf{8 7 . 6}$ & 92.4 & 89.7 & - \\
& Fang et al. [9] & ResNet,90K & 82 & 83.9 & 91.9 & 90.7 & - \\
& Our method & ResNet,90K & $\mathbf{8 4 . 5}$ & 83.3 & $\mathbf{9 3 . 1}$ & $\mathbf{9 2 . 7}$ & 15.6 \\
\hline \multirow{6}{*}{ II } & Shi et al. [46] & VGG,90K+ST & 82.9 & 81.6 & 93.1 & 91.1 & 8.3 \\
& Shi et al. [47]* & VGG,90K+ST & 86.2 & 85.8 & 93.9 & 92.6 & 10.8 \\
& Cheng et al. [5] & ResNet,90K+ST & 87.4 & 85.9 & 94.2 & 93.3 & - \\
& Borisyuk et al. [3] & ResNet,90K+ST & 84.3 & 84.7 & 93.4 & 90,9 & 44.3 \\
& Cheng et al. [6] & ResNet,90K+ST & 83.6 & 84.4 & 91.5 & 90.8 & - \\
& Fang et al. [9] & ResNet,90K+ST & 86.7 & 86.7 & 94.8 & 93.5 & - \\
& Bai et al. [2] & VGG,90K+ST & 85 & 86.2 & 93.7 & 93 & - \\
& Liu et al. [32] & ResNet,90K+ST & 87.0 & 86.9 & 94.4 & 92,8 & 48.7 \\
& Shi et al. [49] & ResNet,90k+ST & $\mathbf{9 3 . 4}$ & $\mathbf{9 3 . 6}$ & 94.5 & 91.8 & - \\
& Luo et al. [37] & -,90K+ST & 91.2 & 88.3 & 95.0 & 92.4 & - \\
& Li et al. [27] & ResNet,90K+ST & 91.5 & 84.5 & - & 91 & - \\
& Liao et al. [28] & VGG,90K+ST & 91.9 & 86.4 & - & 91.5 & - \\
& Wang et al. [55] & ResNet,90K+ST & 93.3 & 88.1 & - & 91.3 & - \\
& Baek et al. [1] & ResNet,90K+ST & 87.9 & 87.5 & 94.9 & 93.6 & 49.6 \\
& Our method & ResNet,90K+ST & 91.4 & 88.8 & $\mathbf{9 5 . 5}$ & $\mathbf{9 4 . 9}$ & 15.6 \\
\hline
\end{tabular}

*means the conference version of [49]. "ST " means including character-level annotations or using private datasets. Type I is unconstrained methods trained on Synth90K (90K). Type II is unconstrained methods trained on Synth90k and SynthText $(90 \mathrm{k}+\mathrm{ST})$.

IIIT5K, SVT, IC03, and IC13, and the irregular datasets include IC15, SVTP, and CUTE. Table 4 and Table 5 show the comparison results on regular datasets and irregular datasets, respectively. We test our method under two different conditions. One uses Synth $90 \mathrm{~K}(90 \mathrm{~K})$ as training datasets, and the other uses Synth90K and SynthText $(90 \mathrm{~K}+\mathrm{ST})$ as training datasets.

Performance on regular datasets. As shown in Table 4, we divide the results of experiment into two categories according to the training datasets we used. From Type I, we can learn that our method achieves the best performance to the existing methods on most of regular datasets except for SVT. Our method gives relative accuracy increases of $1.3 \%$ (from $83.2 \%$ to $84.5 \%$ ) on IIIT5K, $0.7 \%$ on IC03, and $2 \%$ on IC13. As can be seen from Type II, our method achieves highly competitive performance than the state-of-the-art methods. Specifically, our method outperforms all methods on IC03 and IC13 and gives relative accuracy increases of $0.5 \%$ (from $95.0 \%$ to $95.5 \%$ ) and $1.4 \%$, respectively. It is worth noticing that both References [49] and [37] used additional rectification network, which may consume additional computing resources, but our method is not only faster but also more accurate than Reference [37] on all datasets (the speed can be seen in Table 6). Besides, although References [27] and [28] use additional datasets, our method still achieves competitive performance on IIIT5K and SVT. 
Table 5. Irregular Scene Text Recognition Accuracy on Benchmark Datasets

\begin{tabular}{c|c|c|cccc}
\hline Type & Methods & ConvNet,Data & IC15 & SVTP & CUTE & Params $\left(\times 10^{6}\right)$ \\
\hline \multirow{7}{*}{ I } & Shi et al. *[47] & VGG,90K & - & 71.8 & 59.2 & 10.8 \\
& Shi et al. [49] & VGG,90K & 67.6 & 73.2 & 63.9 & \\
& Shi [49] & ResNet,90K & 68.9 & 75.4 & 67.4 & \\
& Fang et al. [9] & ResNet,90K & 64.1 & - & - & \\
& Our method & ResNet,90K & 71.1 & 76.3 & $\mathbf{6 9 . 4}$ & 15.6 \\
\hline \multirow{6}{*}{ II } & Shi et al. *[46] & VGG,90K+ST & 69.4 & 70.0 & 65.5 & 8.4 \\
& Shi et al. *[47] & VGG,90K+ST & 74.5 & 76.2 & 70.4 & 10.8 \\
& Cheng et al. [5] & ResNet,90K+ST & 70.6 & - & - & \\
& Cheng et al. [6] &,$- 90 K+S T^{+}$ & 68.2 & 73 & 76.8 & \\
& Liu et al. [33] & ResNet,90K+ST & 60 & 73.5 & - & \\
& Fang et al. [9] & ResNet,90K+ST & 71.2 & - & - & \\
& Bai et al. [2] & VGG,90K+ST & 73.9 & - & - & \\
& Shi et al. [49] & ResNet,90k+ST & 76.1 & 78.5 & 79.5 & \\
& Luo et al. [37] &,$- 90 K+S T$ & 68.8 & 76.1 & 77.4 & \\
& Liu et al. [32] & ResNet,90K+ST & 76.1 & 77.5 & 71.7 & 48.7 \\
& Liao et al. [28] & VGG,90K+ST & - & - & 79.9 & \\
& Wang et al. [55] & ResNet,90K+ST & 74 & 80.2 & $\mathbf{8 5 . 1}$ & \\
& Borisyuk et al. [3] & ResNet,90K+ST & 71.2 & 73.8 & 69.2 & 44.3 \\
& Baek et al. [1] & ResNet,90K+ST & 77.6 & 79.2 & 74.0 & 49.6 \\
& Our method & ResNet,90K+ST & $\mathbf{7 8 . 6}$ & $\mathbf{8 2 . 8}$ & 77.5 & 15.6 \\
\hline
\end{tabular}

Table 6. Speed (fps) of Different Methods

\begin{tabular}{l|c|c|c}
\hline Methods & Training speed & Inference speed & GPU \\
\hline Cheng et al. [5] & 90 & - & M40 \\
Shi et al. [48] & 160 & 5 & Titan X \\
Cheng et al. [6] & 190 & $630^{*}$ & P40 \\
Shi et al. [49] & 360 & 50 & Titan X \\
Our method & $\mathbf{3 7 0}$ & $\mathbf{9 5}$ & P100 \\
\hline
\end{tabular}

fps means the number of images per second. “*” indicates unknown batch size, "GPU" indicates training network on their GPUs. Note that the FLOPS is P100 $\mathrm{P} 40 \approx \operatorname{Titan} \mathrm{X}>\mathrm{M} 40$.

Performance on irregular datasets. As shown in Table 5, in case of using $90 \mathrm{~K}$ as training dataset, our method also achieves the best performance to existing methods on all irregular datasets. Although Reference [49] used an additional rectification network, our method still outperforms it by a large margin. In case of using $90 \mathrm{~K}$ and ST as training datasets, our method achieves the best performance to the existing methods on two of three irregular datasets. Furthermore, using the same training datasets and backbone, our method gives relative accuracy increases of $2.5 \%$ (from $76.1 \%$ to $78.6 \%$ ) on IC15 and $4.3 \%$ on SVTP. Due to use additional datasets, Reference [27] is better than ours on CUTE, but our method still has a higher average accuracy on all datasets than this work.

From Table 4, we can also learn that the performance of our method improves more on irregular datasets than that on regular datasets. There are three reasons: First, the average accuracy is more than $90 \%$ on regular text, thus the improvement is difficult. For example, our method achieves 


\begin{tabular}{|c|c|c|c|}
\hline Input Images & Ground Truth & Prediction (Res 5 ) & Prediction \\
\hline & west & west & west \\
\hline & played & playd & played \\
\hline & life & life & life \\
\hline & manchester & mausthder & manchester \\
\hline & theater & theater & qneater \\
\hline & swarovski & svaroveki & satrqviki \\
\hline
\end{tabular}

Fig. 4. Selected results on IC 15 and CUTE80. Prediction(Res 5) means using results of using singlegranularity visual representations from Res 5.

$95.1 \%$ accuracy, which is the best result on IC03; however, the best result of other methods on this dataset is $94.8 \%$, so the improvement of our method is very small. Second, the images in irregular datasets are usually curved and arbitrarily oriented, and capturing visual representations of these images at single granularity will result in incomplete representations; while our hierarchical attention network considers visual representations of different granularity, which can work better on irregular datasets. Third, compared to $90 \mathrm{~K}$, ST contains lots of arbitrarily oriented, curved and slant images, which can improve the result on irregular datasets.

Performance on training and inference speed. Table 6 lists the training and inference speed of the current methods. We can learn that the training and inference speed of our recognition network is the fastest. For example, the inference of our proposed network is $95 \mathrm{fps}$, which is almost twice faster that of Reference [49] (50 fps).

In summary, from Tables 4, 5, and 6, we can learn that whatever training datasets are used, our recognition network achieves the comparable or better performance to the existing methods on most datasets under less training and inference time, especially irregular datasets.

\subsection{Limitation of the Network and Applications of LVRM}

For a qualitative comparison, Figure 4 visualizes the recognition results on some images from CUTE80 and IC15. Seen from Figure 4, we can learn that for regular scene text images, recognition network based on Res 5 can recognize correctly, such as "west" and "life." While This network does not work well on images with heavy perspective distortions, such as "played" and "manchester." While, the recognition network based on three-granularity visual representations can recognize correctly on these images. However, text representations from multi- granularity visual representations can also bring noise that makes incorrect prediction, such as "theater."

Regarding the justification of our methods, scene text recognition is just an application of our proposed method. We have verified the effectiveness of our proposed network in image classification task. In the extracted feature maps, our proposed LVRM module can utilize the information of adjacent vectors to represent the current feature vector, which is orthogonal to existing methods. LVRM can be easily plugged into many existing CNN architectures to improve the performance of CNNs. 
Table 7. The Classification Accuracy Cifar-100

\begin{tabular}{c|c|c|c|c}
\hline Dataset & VGG 10 & VGG8-LVRM2 & ResNet34 & ResNet28-LVRM2 \\
\hline CIFAR-100 & 72.67 & 73.18 & 76.45 & 75.94 \\
\hline Params $\left(\times 10^{6}\right)$ & 8.82 & 5.67 & 21.6 & 11.3 \\
\hline
\end{tabular}

We simple architectures of ResNet (ResNet 34) [15] as baseline model and replace two residual blocks with two LVRM modues (ResNet28, LVRM2). We use simple architecture of VGG (VGG 10) [50] as baseline network, which the number of kernels in each convolutional layer is $1 / 4$ of the original layer. Replacing two CNN layers with LVRM, we design a compare network (VGG8LVRM2). The image classification results on Cifar-100 [25] are shown in Table 7. It shows that compared with regular CNN layers, our proposed LVRM can not only obtain comparable knowledge representations, but also reduce the number of learnable parameters by several times.

\section{CONCLUSIONS}

Due to the unconstrained appearance of scene text, it is impossible to capture comprehensive text representations from single-granularity visual representations for scene text recognition. In this work, we present a hierarchical attention network to capture the comprehensive text representations. It consists of several hierarchical attention blocks, and each block contains local visual representation module that is responsible for obtaining accurate description of characters, and decoder module generates a fixed size text representation from the different granularity visual representations. Moreover, we design a scene text recognition architecture based on the multigranularity hierarchical attention network. Extensive experiments over several benchmarks show that the proposed method outperforms the existing recognition methods under shorter training and inference time. In the future, we plan to extend this work to text detection and build a model for end-to-end scene text recognition.

\section{REFERENCES}

[1] Jeonghun Baek, Geewook Kim, Junyeop Lee, Sungrae Park, Dongyoon Han, Sangdoo Yun, Seong Joon Oh, and Hwalsuk Lee. 2019. What is wrong with scene text recognition model comparisons? Dataset and model analysis. In Proceedings of the IEEE/CVF International Conference on Computer Vision. IEEE, 4714-4722. DOI : https://doi.org/10. 1109/ICCV.2019.00481

[2] Fan Bai, Zhanzhan Cheng, Yi Niu, Shiliang Pu, and Shuigeng Zhou. 2018. Edit probability for scene text recognition. In Proceedings of the IEEE Conference on Computer Vision and Pattern Recognition (CVPR'18).

[3] Fedor Borisyuk, Albert Gordo, and Viswanath Sivakumar. 2018. Rosetta: Large scale system for text detection and recognition in images. In Proceedings of the 24th ACM SIGKDD International Conference on Knowledge Discovery \& Data Mining, Yike Guo and Faisal Farooq (Eds.). ACM, 71-79. DOI : https://doi.org/10.1145/3219819.3219861

[4] Antoni Buades, Bartomeu Coll, and J.-M. Morel. 2005. A non-local algorithm for image denoising. In Proceedings of the IEEE Conference on Computer Vision and Pattern Recognition (CVPR'05). 60-65.

[5] Zhanzhan Cheng, Fan Bai, Yunlu Xu, Gang Zheng, Shiliang Pu, and Shuigeng Zhou. 2017. Focusing attention: Towards accurate text recognition in natural images. In Proceedings of the IEEE International Conference on Computer Vision (ICCV'17). 5086-5094.

[6] Zhanzhan Cheng, Yangliu Xu, Fan Bai, Yi Niu, Shiliang Pu, and Shuigeng Zhou. 2018. AON: Towards arbitrarily oriented text recognition. In Proceedings of the IEEE Conference on Computer Vision and Pattern Recognition (CVPR'18). 5571-5579.

[7] Navneet Dalal and Bill Triggs. 2005. Histograms of oriented gradients for human detection. In Proceedings of the IEEE Computer Society Conference on Computer Vision and Pattern Recognition. IEEE Computer Society, 886-893.

[8] Yann N. Dauphin, Angela Fan, Michael Auli, and David Grangier. 2017. Language modeling with gated convolutional networks. In Proceedings of the International Conference on Machine Learning (ICML'17). 
[9] Shancheng Fang, Hongtao Xie, Zheng-Jun Zha, Nannan Sun, Jianlong Tan, and Yongdong Zhang. 2018. Attention and language ensemble for scene text recognition with convolutional sequence modeling. In Proceedings of the International Conference on Multimedia (ACM MM'18).

[10] Jianlong Fu, Heliang Zheng, and Tao Mei. 2017. Look closer to see better: Recurrent attention convolutional neural network for fine-grained image recognition. In Proceedings of the IEEE Conference on Computer Vision and Pattern Recognition (CVPR'17). 4438-4446.

[11] Hongchao Gao, Xi Wang, Yujia Li, Jizhong Han, Songlin Hu, and Ruixuan Li. 2019. Self-representation convolutional neural networks. In Proceedings of the IEEE International Conference on Multimedia and Expo. IEEE, 1672-1677.

[12] Jonas Gehring, Michael Auli, David Grangier, Denis Yarats, and Yann N. Dauphin. 2017. Convolutional sequence to sequence learning. In Proceedings of the 34th International Conference on Machine Learning (Proceedings of Machine Learning Research), Doina Precup and Yee Whye Teh (Eds.), Vol. 70. PMLR, 1243-1252. Retrieved from http:// proceedings.mlr.press/v70/gehring17a.html.

[13] Alex Graves, Santiago Fernández, Faustino Gomez, and Jürgen Schmidhuber. 2006. Connectionist temporal classification: Labelling unsegmented sequence data with recurrent neural networks. In Proceedings of the International Conference on Machine Learning (ICML'06).

[14] Ankush Gupta, Andrea Vedaldi, and Andrew Zisserman. 2016. Synthetic data for text localisation in natural images. In Proceedings of the IEEE Conference on Computer Vision and Pattern Recognition (CVPR'16). 2315-2324.

[15] Kaiming He, Xiangyu Zhang, Shaoqing Ren, and Jian Sun. 2016. Deep residual learning for image recognition. In Proceedings of the IEEE Conference on Computer Vision and Pattern Recognition (CVPR'16). 770-778.

[16] M. Jaderberg, K. Simonyan, et al 2015. Spatial transformer networks. In Proceedings of the International Conference on Advances in Neural Information Processing Systems (NIPS'15). 2017-2025.

[17] Max Jaderberg, Karen Simonyan, Andrea Vedaldi, and Andrew Zisserman. 2015. Deep structured output learning for unconstrained text recognition. In Proceedings of the 3rd International Conference on Learning Representations, Yoshua Bengio and Yann LeCun (Eds.). http://arxiv.org/abs/1412.5903.

[18] Max Jaderberg, Karen Simonyan, Andrea Vedaldi, and Andrew Zisserman. 2016. Reading text in the wild with convolutional neural networks. Int. f. Comput. Vis. 116, 1 (2016), 1-20. DOI : https://doi.org/10.1007/s11263-015-0823-z

[19] Max Jaderberg, Andrea Vedaldi, and Andrew Zisserman. 2014. Deep features for text spotting. In Proceedings of the European Conference on Computer Vision (ECCV'14). 512-528.

[20] Tianming Jiang, Jiangfeng Zeng, Ke Zhou, Ping Huang, and Tianming Yang. 2019. Lifelong disk failure prediction via GAN-based anomaly detection. In Proceedings of the 37th IEEE International Conference on Computer Design. IEEE, 199-207. DOI : https://doi.org/10.1109/ICCD46524.2019.00033

[21] Dimosthenis Karatzas, Lluis Gomez-Bigorda, Anguelos Nicolaou, Suman Ghosh, Andrew Bagdanov, Masakazu Iwamura, Jiri Matas, Lukas Neumann, Vijay Ramaseshan Chandrasekhar, Shijian Lu, et al. 2015. ICDAR 2015 competition on robust reading. In Proceedings of the International Conference on Document Analysis and Recognition (ICDAR'15). $1156-1160$.

[22] Dimosthenis Karatzas et al. 2013. ICDAR 2013 robust reading competition. In Proceedings of the International Conference on Document Analysis and Recognition (ICDAR'13).

[23] Hua Wang, Ping Huang, Xubin He, Ran Lai, Wenyan Li, Wenjie Liu, Tianming Yang, Ke Zhou, and Si Sun. 2019. Improving cache performance for large-scale photo stores via heuristic prefetching scheme. IEEE Trans. Parallel Distrib. Syst. 30, 9 (2019), 2033-2045.

[24] Diederik P. Kingma and Jimmy Ba. 2015. Adam: A method for stochastic optimization. In Proceedings of the 3rd International Conference on Learning Representations, Yoshua Bengio and Yann LeCun (Eds.). Retrieved from http:// arxiv.org/abs/1412.6980.

[25] Alex Krizhevsky and G. Hinton. 2009. Learning Multiple Layers of Features from Tiny Images. Technical Report. University of Toronto.

[26] C. Lee, S. Osinderoet al. 2016. Recursive recurrent nets with attention modeling for OCR in the wild. In Proceedings of the IEEE Conference on Computer Vision and Pattern Recognition (CVPR'16). 2231-2239.

[27] Hui Li, Peng Wang, Chunhua Shen, and Guyu Zhang. 2019. Show, attend and read: A simple and strong baseline for irregular text recognition. In Proceedings of the 33th AAAI Conference on Artificial Intelligence (AAAI'19).

[28] Minghui Liao, Jian Zhang, Zhaoyi Wan, et al. 2019. Scene text recognition from two-dimensional perspective. In Proceedings of the AAAI Conference on Artificial Intelligence (AAAI'19).

[29] Tsung-Yi Lin, Piotr Dollár, Ross Girshick, Kaiming He, Bharath Hariharan, and Serge Belongie. 2017. Feature pyramid networks for object detection. In Proceedings of the IEEE Conference on Computer Vision and Pattern Recognition (CVPR'17). 2117-2125.

[30] Zhouhan Lin, Minwei Feng, Cícero Nogueira dos Santos, Mo Yu, Bing Xiang, Bowen Zhou, and Yoshua Bengio. 2017. A structured self-attentive sentence embedding. In Proceedings of the 5th International Conference on Learning Representations. Retrieved from https://openreview.net/forum?id=BJC_jUqxe. 
[31] Wei Liu, Dragomir Anguelov, Dumitru Erhan, Christian Szegedy, Scott Reed, Cheng-Yang Fu, and Alexander C. Berg. 2016. SSD: Single shot multibox detector. In Proceedings of the European Conference on Computer Vision (ECCV'16). Springer, 21-37.

[32] Wei Liu, Chaofeng Chen, Kwan-Yee K. Wong, Zhizhong Su, and Junyu Han. 2016. STAR-Net: A SpaTial Attention Residue Network for scene text recognition. In Proceedings of the British Machine Vision Conference, Richard C. Wilson, Edwin R. Hancock, and William A. P. Smith (Eds.). BMVA Press. Retrieved from http://www.bmva.org/bmvc/2016/ papers/paper043/index.html.

[33] Wei Liu, Chaofeng Chen, and Kwan-Yee K Wong. 2018. Char-Net: A character-aware neural network for distorted scene text recognition. In Proceedings of the 32nd AAAI Conference on Artificial Intelligence (AAAI'18).

[34] Yu Liu, Jingkuan Song, Ke Zhou, Lingyu Yan, Li Liu, Fuhao Zou, and Ling Shao. 2019. Deep self-taught hashing for image retrieval. IEEE Trans. Cybern. 49, 6 (2019), 2229-2241. DOI : https://doi.org/10.1109/TCYB.2018.2822781

[35] Jiasen Lu, Jianwei Yang, Dhruv Batra, and Devi Parikh. 2016. Hierarchical question-image co-attention for visual question answering. In Proceedings of the International Conference on Advances In Neural Information Processing Systems (NIPS'16). 289-297.

[36] Simon M. Lucas, Alex Panaretos, Luis Sosa, Anthony Tang, Shirley Wong, Robert Young, Kazuki Ashida, Hiroki Nagai, Masayuki Okamoto, Hiroaki Yamamoto, Hidetoshi Miyao, JunMin Zhu, WuWen Ou, Christian Wolf, Jean-Michel Jolion, Leon Todoran, Marcel Worring, and Xiaofan Lin. 2005. ICDAR 2003 robust reading competitions: Entries, results, and future directions. Int. F. Doc. Anal. Recog. 7, 2-3 (2005), 105-122. DOI : https://doi.org/10.1007/s10032-0040134-3

[37] Canjie Luo, Lianwen Jin, and Zenghui Sun. 2019. MORAN: A multi-object rectified attention network for scene text recognition. Pattern Recog. 90 (2019), 109-118. DOI : https://doi.org/10.1016/j.patcog.2019.01.020

[38] Xiao Ma, Jiangfeng Zeng, Limei Peng, Giancarlo Fortino, and Yin Zhang. 2019. Modeling multi-aspects within one opinionated sentence simultaneously for aspect-level sentiment analysis. Fut. Gen. Comput. Syst. 93 (2019), $304-311$. DOI : https://doi.org/10.1016/j.future.2018.10.041

[39] Krystian Mikolajczyk and Cordelia Schmid. 2004. Scale \& affine invariant interest point detectors. Int. F. Comput. Vis. 60, 1 (2004), 63-86. DOI : https://doi.org/10.1023/B:VISI.0000027790.02288.f2

[40] Anand Mishra, Karteek Alahari, and C. V. Jawahar. 2012. Scene text recognition using higher order language priors. In Proceedings of the British Machine Vision Conference (BMVC'12).

[41] Anand Mishra, Karteek Alahari, and C. V. Jawahar. 2012. Top-down and bottom-up cues for scene text recognition. In Proceedings of the IEEE Conference on Computer Vision and Pattern Recognition (CVPR'12), 2687-2694.

[42] Volodymyr Mnih, Nicolas Heess, Alex Graves, et al. 2014. Recurrent models of visual attention. In Proceedings of the International Conference on Advances in Neural Information Processing Systems (NIPS'14). 2204-2212.

[43] Timo Ojala, Matti Pietikäinen, and Topi Mäenpää. 2002. Multiresolution gray-scale and rotation invariant texture classification with local binary patterns. IEEE Trans. Pattern Anal. Mach. Intell. 24, 7 (2002), 971-987. DOI : https://doi. org/10.1109/TPAMI.2002.1017623

[44] Trung Quy Phan, Palaiahnakote Shivakumara, Shangxuan Tian, and Chew Lim Tan. 2013. Recognizing text with perspective distortion in natural scenes. In Proceedings of the IEEE International Conference on Computer Vision (ICCV'13). 569-576.

[45] Anhar Risnumawan, Palaiahnakote Shivakumara, Chee Seng Chan, and Chew Lim Tan. 2014. A robust arbitrary text detection system for natural scene images. Exp. Syst. Appl. 41, 18 (2014), 8027-8048. D0I : https://doi.org/10.1016/j. eswa.2014.07.008

[46] Baoguang Shi, Xiang Bai, and Cong Yao. 2017. An end-to-end trainable neural network for image-based sequence recognition and its application to scene text recognition. IEEE Trans. Pattern Anal. Mach. Intell. 39, 11 (2017), 22982304. DOI : https://doi.org/10.1109/TPAMI.2016.2646371

[47] Baoguang Shi, Xinggang Wang, Pengyuan Lyu, Cong Yao, and Xiang Bai. 2016. Robust scene text recognition with automatic rectification. In Proceedings of the IEEE Conference on Computer Vision and Pattern Recognition (CVPR'16), 4168-4176.

[48] Baoguang Shi, Xinggang Wang, Pengyuan Lyu, Cong Yao, and Xiang Bai. 2016. Robust scene text recognition with automatic rectification. In Proceedings of the IEEE Conference on Computer Vision and Pattern Recognition (CVPR'16).

[49] Baoguang Shi, Mingkun Yang, Xinggang Wang, Pengyuan Lyu, Cong Yao, and Xiang Bai. 2019. ASTER: An attentional scene text recognizer with flexible rectification. IEEE Trans. Pattern Anal. Mach. Intell. 41, 9 (2019), 2035-2048. DOI : https://doi.org/10.1109/TPAMI.2018.2848939

[50] Karen Simonyan and Andrew Zisserman. 2015. Very deep convolutional networks for large-scale image recognition. In Proceedings of the 3rd International Conference on Learning Representations, Yoshua Bengio and Yann LeCun (Eds.). Retrieved from http://arxiv.org/abs/1409.1556.

[51] Zhuotao Tian, Michelle Shu, Pengyuan Lyu, Ruiyu Li, Chao Zhou, Xiaoyong Shen, and Jiaya Jia. 2019. Learning shape-aware embedding for scene text detection. In Proceedings of the IEEE Conference on Computer Vision and Pattern Recognition (CVPR'19). 4234-4243. 
[52] Ashish Vaswani, Noam Shazeer, Niki Parmar, Jakob Uszkoreit, Llion Jones, Aidan N. Gomez, Lukasz Kaiser, and Illia Polosukhin. 2017. Attention is all you need. In Proceedings of the International Conference on Advances in Neural Information Processing Systems (NIPS'17). 6000-6010.

[53] Jianfeng Wang and Xiaolin Hu. 2017. Gated recurrent convolution neural network for OCR. In Proceedings of the International Conference on Advances in Neural Information Processing Systems (NIPS'17). 334-343.

[54] Kai Wang, Boris Babenko, and Serge Belongie. 2011. End-to-end scene text recognition. In Proceedings of the IEEE International Conference on Computer Vision (ICCV'11).

[55] Peng Wang, Lu Yang, et al. 2019. A simple and robust convolutional-attention network for irregular text recognition. In Proceedings of the AAAI Conference on Artificial Intelligence (AAAI'19).

[56] Xiaolong Wang, Ross B. Girshick, Abhinav Gupta, and Kaiming He. 2018. Non-local neural networks. In Proceedings of the IEEE Conference on Computer Vision and Pattern Recognition. IEEE Computer Society, 7794-7803.

[57] X. Yang, D. He, et al. 2017. Learning to read irregular text with attention mechanisms. In Proceedings of the International foint Conferences on Artificial Intelligence Organization (IfCAI'17). 3280-3286.

[58] Cong Yao, Xiang Bai, Baoguang Shi, and Wenyu Liu. 2014. Strokelets: A learned multi-scale representation for scene text recognition. In Proceedings of the IEEE Conference on Computer Vision and Pattern Recognition (CVPR'14). 40424049 .

[59] Qixiang Ye and David Doermann. 2015. Text detection and recognition in imagery: A survey. IEEE Trans. Pattern Anal. Mach. Intell. 37, 7 (2015), 1480-1500.

[60] Jiangfeng Zeng, Xiao Ma, and Ke Zhou. 2019. Enhancing attention-based LSTM with position context for aspect-level sentiment classification. IEEE Access 7 (2019), 20462-20471. DOI : https://doi.org/10.1109/ACCESS.2019.2893806

[61] Jiangfeng Zeng, Xiao Ma, and Ke Zhou. 2019. Photo-realistic face age progression/regression using a single generative adversarial network. Neurocomputing 366 (2019), 295-304. DOI : https://doi.org/10.1016/j.neucom.2019.07.085

[62] Ji Zhang, Yu Liu, Ke Zhou, Guoliang Li, Zhili Xiao, Bin Cheng, Jiashu Xing, Yangtao Wang, Tianheng Cheng, Li Liu, Minwei Ran, and Zekang Li. 2019. An end-to-end automatic cloud database tuning system using deep reinforcement learning. In Proceedings of the International Conference on Management of Data, Peter A. Boncz, Stefan Manegold, Anastasia Ailamaki, Amol Deshpande, and Tim Kraska (Eds.). ACM, 415-432. DOI : https://doi.org/10.1145/3299869. 3300085

[63] Ke Zhou, Jiangfeng Zeng, Yu Liu, and Fuhao Zou. 2018. Deep sentiment hashing for text retrieval in social CIoT. Fut. Gen. Comput. Syst. 86 (2018), 362-371. DOI : https://doi.org/10.1016/j.future.2018.03.047

Received March 2020; revised December 2020; accepted January 2021 